\title{
CHEMICAL ROUTES TO DUST FORMATION IN THE EJECTA OF NOVAE
}

J.M.C. Rawlings, D. A. Williams

Mathematics Department, UMIST, P.O. Box 88, Manchester, M60 1QD, U.K.

The chemistry of the formation of nucleation sites in the ejecta of novae has been investigated. Previous authors (e.g. Clayton and Wickramasinghe 1976) have noted that grain growth is rapid once nucleation sites have formed but the assumption is generally made that the ejecta is in LTE during the nucleation phase. This is obviously not the case as the ejecta is illuminated by an intense radiation field so that the lifetimes of the larger species are of the order of one second, even the ejecta kinetic temperature is only 1000-2000 K. We propose that nucleation sites form by heterogeneous chemical reactions in a highly non-equilibrium chemistry.

A kinetic chemical model has been developed which uses the results from previous work (Rawlings 1986) as a starting point in the calculations. In that work it was shown that in a CII region the effects of coulombic repulsion, the unshielded UV radiation field and the high temperature strongly inhibit chemistry and the formation of nucleation sites. In a CI region the lower temperature and the blocking provided by the carbon continuum result in the formation of significant amounts of $\mathrm{H}_{2}$ and $\mathrm{CO}$ (only) so that, if the temperature is below about $4000 \mathrm{~K}$, the CO saturates. Thus if $\mathrm{C}>0$ in the dust forming part of the ejecta, all of the free oxygen will be locked up in CO. The only viable route leading to the formation of graphitic nucleation sites is via a hydrocarbon chemistry, so that the depletion of 0 into CO (which would otherwise "burn" the hydrocarbons) is essential to the chemistry. The model includes all species up to $\mathrm{C}_{8} \mathrm{H}_{6}$ which represents something of a kinetic bottleneck in the chemistry. Hydrogenation is kept low by the action of the UV radiation field. As a result of the intense $U V$ only reactions involving the smaller, partially shielded species (such as $\mathrm{C}_{2}$ ) are significant in the growth process. Many of the rate co-efficients for reactions involving larger species are unknown and have been estimated by extrapolating trends in similar reactions for smaller species.

For FH Ser (1970) data implies that if all of the carbon is depleted into grains the minimum fractional abundance of sites required is only $10^{-15}$ to $10^{-14}$. Results from the model show that $\mathrm{H}_{2}$ formation is very slow indeed and that the efficiency of the chemistry depends on the presence of significant quantities of pre-existent $\mathrm{H}_{2}$. Key species in the scheme are: $\mathrm{H}_{2}, \mathrm{C}_{2}^{(+)}, \mathrm{C}^{(+)}, \mathrm{CH}^{(+)}, \mathrm{C}_{2} \mathrm{H}^{(+)}$. The chemistry is insensitive to parameter variations with the exception of the density, the fractional ionization and the fractional abundance of $\mathrm{H}_{2}$ at the time of the start of the calculations (30 
days) to which it is very sensitive. It is found that fractional abundances of $10^{-15}$ for the largest species can be achieved if the initial $\mathrm{H}_{2}$ abundance is greater than $10^{-3}$ and the fractional ionization is less than $10^{-3}$.

The main conclusions of this work are:

(i) Dust can only form in a CI region (where LTE does not prevail).

(ii) The locking up of all free oxygen in $\mathrm{CO}$ is essential to the chemistry. Dust can only form in a region where $C>0$.

(iii)Only reactions with small species (eg, $\mathrm{C}_{2}$ ) are important. $\mathrm{H}_{2}$ is rapidly absorbed by the the chemistry so that rapid grain formation is necessary so as to take up the available nucleation sites before they are destroyed by the UV radiation field.

(iv) In order to create the required number of nucleation sites high $\mathrm{H}_{2}$ fractional abundances and low ionization levels are required. However, if $\mathrm{S}, \mathrm{Si}, \mathrm{Mg}$ or $\mathrm{Fe}$ are neutral, flux shielding severely inhibits the ion-molecule chemistry to such an extent that nucleation sites cannot be formed. This may explain why HR Del (which had a very low ionization level) failed to produce any dust despite having a very massive ejecta. To obtain the required $\mathrm{H}_{2}$ abundance either,

a) The ejecta temperature at $t=30$ days must be $<1500 \mathrm{~K}$, falling slowly, or,

b) The density of the ejecta in the dust-forming region must be $>10-100 \mathrm{x}$ the mean ejecta value. This could be achieved if the dust-forming region is a thin dense shell of material at the edge of the ejecta. This shell must be stable against breaking up or else dust formation would not 'blacken the sky' of the nova and a deep transition would not be observed.

(v) It is expected that the ratio of nucleation sites to grains will be of the order of unity. Since the total number of grains in the ejecta is roughly $10^{38}$ this corresponds to a column density of nucleation sites of just $10^{10} \mathrm{~cm}^{-2}$. It is therefore hardly surprising that molecular emission lines (other than $\mathrm{CO}$ ) have not been detected, especially as the lifetimes of some of the intermediate size species are of the order of seconds.

(vi) The likelihood of the formation of dust in the ejecta of a nova is primarily determined by the ejecta's configuration, elemental composition and ionization structure.

References:

Clayton, D. D. and Wickramasinghe, N. C. , 1976, Ast.Sp. Sci., 42, 463.

Rawlings, J.M.C., 1986, Mon. Not.R. astr.Soc., 232, 507. 